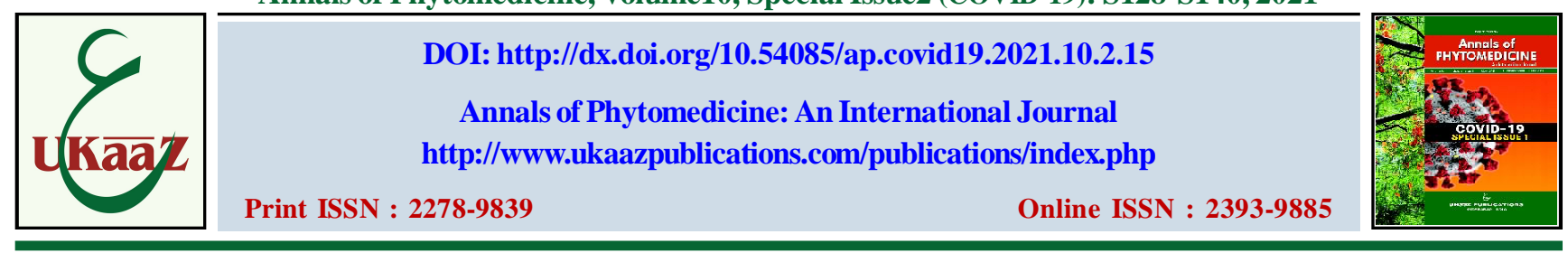

\title{
Role of wild fruits in combating COVID-19 infection: An overview
}

\author{
Kanchan Bhatt, Sunakshi Gautam», Abhimanyu Thakur, N.S. Thakur, Hamid* and Kritika Kaushal \\ Department of Food Science and Technology, Dr. Yashwant Singh Parmar University of Horticulture and Forestry, Nauni, Solan-173230, \\ Himachal Pradesh, India \\ *Food Technology and Nutrition, School of Agriculture, Lovely Professional University, Phagwara, Punjab-144411, India
}

\begin{tabular}{|c|c|}
\hline Article Info & Abstract \\
\hline Article history & \multirow{12}{*}{$\begin{array}{l}\text { COVID-19, a disease caused by SARS-COV-2 virus was the reason for the pandemic and loss of many lives. } \\
\text { The virus transmission from human-to-human touch led to outbreaks of this disease. World health } \\
\text { organization has recommended a list of precautionary measures to avoid the major infection of this } \\
\text { virus. This list includes various medicinal plants, fruits and vegetables which can boost the immunity } \\
\text { and help in reducing the risk of infection. Due to this COVID-19, the food habits of most of the people } \\
\text { changed which led to shift towards healthier lifestyle. As most of the Indian population is vegetarian, } \\
\text { so the health based plant food including wild plant sources have been explored extensively. The wild } \\
\text { fruits like bael, aonla, jamun, rubus, aakhe, simul and many more have the antiviral properties and } \\
\text { immune boosting properties which could be incorporated in our diet. The bioactive compounds like } \\
\text { polyphenolics, flavonoids, tannins, phytosterols, vitamins, minerals, etc., present in above mentioned } \\
\text { wild fruits can be utilized to have healthful benefits. As these functional components can help in } \\
\text { reducing various diseases like asthma, bronchitis, cold, fever, flu, inflammatory diseases, cancer, } \\
\text { cardiovascular diseases and ultimately boost up the immunity to cope up with CovID-19 illness. }\end{array}$} \\
\hline Received 25 October 2021 & \\
\hline Revised 13 December 2021 & \\
\hline Accepted 14 December 2021 & \\
\hline Published Online 30 December 2021 & \\
\hline Keywords & \\
\hline COVID-19 & \\
\hline Diseases & \\
\hline Immunity & \\
\hline Health & \\
\hline Phytochemicals & \\
\hline Wild fruits & \\
\hline
\end{tabular}

\section{Introduction}

COVID-19 a disease caused by member of Coronaviridae family, subfamily Orthocoronavirinae, order Nidovirales commonly known as SARS-CoV-2 and have unique surface projections/spikes from where it gets its name, i.e., corona which means crown in Latin. The symptoms of COVID-19 are similar to severe acute respiratory syndrome (SARS), Middle East respiratory syndrome (MERS) and common cold (Shaikh et al., 2021). The emergence of COVID-19 as a pandemic was reported on December, 2019, in Wuhan China (Wiersinga et al., 2019). Originally, the initial spread has started by zoonotic transmission from seafood market of Wuhan, China was considered as the major reason for outbreak. However, later the human-to-human transmission was considered as major cause of its uncontrolled outbreak (Yuki et al., 2020). The most prominent symptoms of infections of SARS virus are respiratory symptoms such as cough, breathing problems, fever and in more severe cases, it led to acute respiratory syndrome, pneumonia and sometime death (WHO, 2020). Besides these symptoms, it also affects nervous, cardiovascular and gastrointestinal system of human body (Monnerat et al., 2020). The individual factors which plays a crucial role in the severity of infection are age of the person, social conditions, ethnicity, nutritional status, malnutrition, pre-existing health conditions and most important the immunity status (Sooriyaarachchi et al., 2021).

Corresponding author: Ms. Sunakshi Gautam Department of Food Science and Technology, Dr. Yashwant Singh Parmar University of Horticulture and Forestry, Nauni, Solan-173230, Himachal Pradesh, India

E-mail: sunakshigautam7@gmail.com

Tel.: +91-9805588330

Copyright () 2021 Ukaaz Publications. All rights reserved.

Email: ukaaz@yahoo.com; Website: www.ukaazpublications.com
Food plays a very important role in prevention of infection of coronavirus by enhancing the immunity by providing various phytonutrients and bioactive components. According to the report of WHO (2020), bioactive compounds obtained from natural food sources have the capability to lower the risk of this deadly disease. The presence of flavonoids, polyphenols, sterols, alkaloids, terpenoids, vitamins, minerals, unsaturated fatty acid and micronutrients which are abundantly present in fruits and vegetables helps in prevention and control of this deadly viral infection in human body (Hamid et al., 2021). These bioactive functional components can be used as therapeutic agents against SARS-CoV-2 or help in preventive activities of COVID patients (Monnerat et al., 2020). The phytoconstituents like polyphenols, flavonoids and tannins are very essential as they act as antioxidants and antiinflammatory agents and regulate the immune cells as well as gene expressions (Monnerat et al., 2020). The functioning of immune system depends on the nutritional status of body and interaction among different food components (Thakur et al., 2019). It has been observed that due to spread of this deadly virus, the eating pattern, habits, healthy-unhealthy food ratio changed very drastically and people are becoming more inclined towards immune boosting healthy foods (Kutyauripo et al., 2021).

Although, major proportion of world's population is getting vaccinated and the chances of this deadly disease is minimized by taking care of precautionary measures. But, due to changes in its genetic makeup and mutation, this virus can still cause harm to human race. So, it become as very important to maintain health and immunity to fight against virus/disease. According to World health organization report, about $80 \%$ of total world population rely on vegetarian diet to fulfill their nutritional requirement as plant possess bioactive compounds with antivairal and anti-inflammatory activities (Ashraf et al., 2021). In India, the wide plant diversity makes it possible to ensure better health. The country occupies $2 \%$ of earth 
surface consists of 5\% world's diversity and known as a hotspot for biodiversity (Sasi and Rajendran, 2012). There are various wild fruits species available in various geographical areas of India which can be helpful in prevention of future outbreaks of these kind of diseases. The wild flora and fauna of India containing high concentration of bioactive compounds and nutritional components, which can directly or indirectly help in prevention of COVID-19 infection. So, by keeping in mind the symptoms caused by SARS-CoV-2 and its impact on different body parts; this review covers the role of wild fruits and their bioactive components which can help in prevention of this deadly virus.

\section{Wild fruit species available in India}

Wild fruits are very important for villagers and farmers as they are the chief source of food as well as for economic aspects for their livelihood (Mahapatra et al., 2012). India is the natural habitat of many species of fruits with therapeutic and medicinal properties. The fruit species like Carissa spinarum, Opuntia sp., Syzygium cumunii, Aegle marmelos and many other are available in central parts of India throughout the year, whereas, Artocarpus laucha, Physalis peruviana, Diospyros sp. are prevalent in western and northern sides (Ahirvar et al., 2017; Biswas et al., 2018). North and South parts of India are known for wild fruit species like Ficus palmate, Elaeagnus umbellate, Morus alba, Phyllanths emblica, Ziziphus mauritiana, Opuntia sp., etc. (Kumar and Shiddamallayya, 2016; Bhatia et al., 2018). All over India, there is abundance of wild fruit species having high nutritional as well as medicinal value and there is wide scope of their utilization in curing many diseases which needs to be explored. The list of some important wild fruits commonly consumed in India has been presented in Table 1.

Table 1: Different wild fruit species available in India

\begin{tabular}{|c|c|c|c|c|}
\hline Sr. No. & Wild fruit & Common name & Family & References \\
\hline 1. & Aegle marmelos & Bael & Rutaceae & He et al., 2016; \\
\hline 2 & Artocarpus laucha & Dheu, Monkey jack, Lakuchi & Moraceae & Younus et al., 2016; \\
\hline 3 . & Baccaurea sapida & Bermese grape & Euphorbiaceae & Ota et al., 2016; \\
\hline 4. & Berberis aristata & Kashmal, Indian barberry, Tree turmeric, Daruharidra & Berberidaceae & Gogoi, 2017; \\
\hline 5 . & Bombax ceiba & Silk cotton tree, Simal, Semul, Shalmali & Bombacaceae & Hamid et al., 2017; \\
\hline 6. & Carissa spinarum & Karondhu, Kharnu, Garna & Apocynaceae & Kaunda and Zhang, 2017; \\
\hline 7. & Cordia dichotoma & Bhokar, Lasuda, Indian cherry, Gondi & Boraginaceae & Prajapati et al., 2017; \\
\hline 8. & Cornus capitate & Himalayan strawberry tree, Tharbal & Cornaceae & Thakur et al., 2017; \\
\hline 9 & Crataegus songarica & Bansangli & Rosaceae & Manandhar et al., 2018; \\
\hline 10 & Diospyros lotus & Wild persimmon & Ebenaceae & Bhat et al., 2018; \\
\hline 11. & Elaeagnus umbellate & Ghain, Chndar, Bastard oleaster & Elaeagnacea & Neag et al., 2018; \\
\hline 12. & Ficus auriculata & Timble, Timla, Timble, Gular & Moraceae & Kabra et al., 2019; \\
\hline 13. & Ficus palmate & Anjiri, Bedu, Khemri & Moraceae & Sharma et al., 2019; \\
\hline 14. & Juglans regia & English or Persian walnut & Juglandaceae & Bhatt et al., 2020; \\
\hline 15. & Morus alba & White mulberry, Shehtoot & Moraceae & Murathan, 2020; \\
\hline 16. & Myrica esculenta & Kaiphal or katphal & Myricaceae & Zulfqar et al., 2020; \\
\hline 17. & Phyllanths emblica & Amla, Indian gooseberry & Phyllanthaceae & Hamid et al., 2020; \\
\hline 18 & Physalis peruviana & Rasbhary, Cape gooseberry & Solanaceae & Sahu et al., 2020; \\
\hline 19. & Pinus gerardiana & Chilgoza & Pinaceae & Thakur et al., 2020; \\
\hline 20. & Prunus americana & Chulli, Chulu & Rosaceae & Pal, 2020; \\
\hline 21. & Punica granatum & Daadu & Punicaceae & Ahmad et al., 2021; \\
\hline 22 . & Rubus ellipticus & Yellow Himalayan Raspberry, Hisalu & Rosaceae & Tamta et al., 2021; \\
\hline 23. & Ziziphus mauritiana & Ber, Chinese date & Rhamnaceae & Soni and Malik, 2021 \\
\hline 24. & Syzygium cumunii & Jamun, Java plum, Indian blackberry & Myrtaceae & \\
\hline 25 . & Celtis australis & $\begin{array}{l}\text { Mediterranean hackberry, European nettle tree, or } \\
\text { the lote tree }\end{array}$ & Cannabaceae & \\
\hline 26. & Malus baccata & Siberian crab apple & Rosacea & \\
\hline 27. & Opuntia dillenii & Wild prickly pear & Cactaceae & \\
\hline
\end{tabular}




\section{Wild fruits and COVID-19}

Wild fruits are the rich source of nutrients and more concentrated source of bioactive components and act as healthy therapeutic alternative because of their better tolerance in human body as they are derived from natural sources (Singh et al., 2021). The functional components present in these fruits can be used for positive pharmacological effects and have the potential to act as antiviral agent and boost the immunity (Hamid et al., 2021). The fruits and their bioactive components have been reported for their use in quenching respiratory diseases, viral infections, immune boosting properties, regulation of natural processes in human body and other health related effects associated to COVID-19 infection. The various fruit which can directly or indirectly helps in preventing/reducing the risk of COVID-19 has been enlisted in Table 2 and the role of some of the important wild fruits in preventing COVID-19 illness has been explained further.

\subsection{Bael (Aegle marmelos)}

Bael fruits are known to effectively reduce the respiratory diseases, thus can prevent the infection of lungs and COVID-19 (Yadav et al., 2020). The various phytochemicals present in its fruit act as anticoronavirus agent and play a crucial role in quenching various other diseases (Khadka et al., 2021). The presence of marmele/marmelide $\left(\mathrm{C}_{16} \mathrm{H}_{14} \mathrm{O}_{4}\right)$ compound in bael fruit is responsible for its various antiviral properties. This component influences the early stage of replicative cycle of viruses such as adsorption, penetraton, etc. (Maity et al., 2009) and blocks the receptors site for virus binding (Santhi et al., 2021). It also block the replication of genetic material of virus replication which led to minimize the contact with host and does not allow it to show cytotoxicity. Mermelide showed the similar results as that of ribavirin which is a popular virucidal and chemotherapeutic agent (Yadevendra et al., 2020) and exhibits antimalarial activity by suppressing the development of Plasmodium falciparum. The higher concentration of vitamin $\mathrm{C}$ helps in lowering the effect of H1N1 (swine flu) and CV-B3 virus titers by relieving lung irritation and respiratory illness (Yadav et al., 2020; Santhi et al., 2021).

\subsection{Aonla (Phyllanthus emblica)}

The fruits of aonla are used in Indian as well as Unani medicine system due to abundance of phytochemicals present in it. In relation to COVID-19, the phytoconstituents like ascorbic acid, phyllaemblicin, phyllaemblinol and 1,2,4,6-tetra-O-galloyl- $\beta$-Dglucose plays an important role in preventing its infection. Phyllaemblicin G7 and B and phyllaemblinol are reported to suppress the viral activity in COVID-19 (Khadka et al., 2021). These are helicase inhibitor which does not allow the virus of COVID to replicate as helicase is the key protein and essential for viral growth (Singh $e t$ $a l ., 2021)$. Whereas 1,2,4,6-tetra-O-galloyl- $\beta$-D-glucose is a polyphenolic compound present in aonla fruits which act as a potential antiviral agent works against HS virus by inhibition of penetration, suppression of intracellular growth, thus suppressing early infection and inhibition of viral biosynthesis in host cells (Gyawali et al., 2020; Ahmed et al., 2021). Besides these health benefits, the other components present in aonla fruit like geraniin and isocorilagin have immunostimulatory effects and helps in boosting the immune system (Ahmed et al., 2021).

\subsection{Box myrtle (Myrica esculenta)}

In box myrtle (kaphal) fruits, myricitrin a glycosylated analog of myricetin has been found effective for the prevention of Ebola virus, HIV and SARS coronavirus (Joshi et al., 2021). Also, myricitrin have higher binding affinity as compared to myricetin and it inhibit the helicase protein which is essential protein for SARS virus for ATPase activity and replicate its genetic material, thus can help in reducing the risk of COVID-19 (Patel et al., 2021).

\subsection{Dheu (Artocarpus laucha)}

The fruits of Atrocarpus lakucha are rich in various flavonoids and triterpenoids and prevent various viral diseases as well as malaria. The flavonoids block the transcription and translation process in virus and does not allow it to replicate, thus does not allow the infection causing virus to get attached with host cells (Buddhisuharto et al., 2021).

\subsection{Dharu/wild pomegranate (Punica granatum)}

The fruits of dharu are rich source of anthocyanins and ellagitannins, more specifically punicalagins and its derivatives. These components are responsible for the antiviral properties of this fruit and they prevent binding, penetration, cell-to-cell infection and secondary infection (Sallese et al., 2021). Also, punicalagin has been reported as the major component to suppress the activity of influenza virus and showed synergistic effects with oseltamivir which is a influenza curing drug (Sallese et al., 2021). Zivkovic et al. (2021) have reported the juice and peel extract was reported to be beneficial for preventing human noroviruses (HuNoV). In other studies on COVID/ SARS, the components like ellagic acid, gallic acid and quercetin exhibited antiviral activity against its viral infection and these compounds are abundantly present in P. granatum fruits. Pomegranate extract has also been used effectively against herpes, influenza and human immunodeficiency virus (Shaygannia et al., 2015).

\subsection{Prickly pear (Opuntia dillenii)}

The fruits of prickly pear contain $\beta$-sitosterol and $\beta$-glucuronidase, which act as anti-inflammatory agents and also effective against herpesvirus, coronavirus and other viral diseases (Shin et al., 2004).

\subsection{Ber (Ziziphus mauritiana)}

Ber fruits are used in Persian, Chinese and Korean system of medicine since ancient time. Fruits of Ziziphus sp. has been used for curing the chest complaints and other related diseases (Goyal et al., 2012). The components like triterpenic and betulinic acid are the active ingredient responsible for immune boosting property, whereas, betulinic acid and Jujuboside B are responsible for curing the various cardiovascular diseases (Shahrajabian et al., 2019).

\subsection{Rasbhary/cape gooseberry (Physalis peruviana)}

The fruits of cape gooseberry has been reported to be effective for the treatment of asthma, malaria, sore throat, eye infection and act as immunity booster (Kasali et al., 2021).

\subsection{Chilgoza (Pinus gerardiana)}

The nuts of chilgoza are very effective antimicrobial (antiviral, antifungal, antibacterial) and antiseptic agent and helps in regulating the blood pressure. Also, the antidiabetic component of this fruit is responsible for inhibiting the $\alpha$-amylase activity, thus helpful in reducing cardiovascular diseases (Zulfqar et al., 2019). The 
sesquiterpenes are responsible for antiseptic property of the nut, whereas, flavan-3-ols and benzoic acid helps in inhibition of $\alpha$-amylase activity and decrease the blood glucose level (Bhardwaj et al., 2021).

\subsection{Daru haldi (Berberois aristata)}

Berberine, a phytochemical present in $B$. aristata showed the effective results for the treatment of various viral diseases like malaria and fever and also cure diabetes (Neag et al., 2018).

\subsection{Bushplum/Kurundhu (Carissa spinarum)}

The phytochemicals (flavonoids, alkaloids, alanine, etc.) present in the fruit of $C$. spinarum showed the effectivity against fever, sore throat, malaria and antiviral activity (Fatima et al., 2013).

\subsection{Simul (Bombax ceiba)}

The presence of phytochemicals in simul fruit, the extract of $B$. ceiba fruits showed hypotensive, hypoglycaemic and antioxidant activity as well used as an anti-inflammatory agent and analgesic (Jalalpure and Gadge, 2011). Also, due to the presence of higher concentration of phenolic compounds and flavonoids, it helps in neutralizing the free radicals like $\mathrm{H}_{2} \mathrm{O}_{2}$ and protect the cells from hemolysis (Divya et al., 2012).

\subsection{Ghain/Chndar (Elaeagnus umbellate)}

The fruits of ghain/bastard oleaster are rich in carotenoids (lycopene, $\alpha$ and $\beta$-cryptoxanthin, lutein, $\beta$-carotene, phytofluene and phytoene), flavonoids, monoterpenes, organic acids and vitamin $\mathrm{C}$ and oils (vitamin $\mathrm{E}$ and phytosterol). This oil/phytosterols are used to cure various heart diseases as its anticoagulant property helps in lowering the blood cholesterol (Ahmad et al., 2005). These barriers are known to prevent the chain reactions, neutralizing short-lived oxidative damage prevent deterioration of tissues and can cure cancer, immunological activity, cardiovascular diseases and degenerative diseases (Ozen et al., 2017). The anticancerous properties and high singlet oxygen quenching ability of its fruit are used to cure diabetes and inflammations (Gamba et al., 2020).

\subsection{Anjiri/Bedu/Khemri (Ficus palmate)}

The fruits of $F$. palmate are very effective against lung diseases and hypoglycemia, gastrointestinal disorders, ulcer, tumour, hyperlipidemia, diabetes and fungal infections (Joshi et al., 2014). Since the ancient times, the fruit extract of wild figs has been used to cure respiratory diseases like sore throats, cough, bronchial problems, etc., and used as an demulcent, emollient, laxative and poultice agent (Rusmadi et al., 2020).

\subsection{Aakhe (Rubus ellipticus)}

The presence of flavonoids in aakhe fruits make it an important antioxidant with high redox potential. Its components can effectively scavenge reactive oxygen species and bind free radicals in the human body (Cao et al., 1997). Flavonoids and related components act as anti-inflammatory, antihepatotoxic, antiulcer, antiallergic, antiviral and anticancerous agent (Umamaheswari and Chatterjee, 2008).

\subsection{Jamun (Syzygium cumunii)}

The fruits of jamun are well known for its use in curing diabetes, malaria, cough, cold, bronchitis, etc. This fruit has antibacterial, antiviral, antifungal as well as antioxidant properties (Sahu et al., 2020). Antidiabetic properties of it are due to the presence of glycoside, jamboline and ellagic acid which controls blood glucose level (Giri et al., 1985). It was evident that people suffering from diabetes were more prone to COVID-19, thus jamun can really help the diabetic patients in lowering down their sugar level. This fruit contains various phytoconstituents like flavonoids, phenols, carotenoids and vitamins, thus are very helpful in lowering down the oxidative stress as well as degenerative disesases (Kubola et al., 2011).

\subsection{Shehtoot/mulberry (Morus alba)}

The fruits of mulberry contain the compound, namely; 1-deoxyno jirimycin which is responsible for its antiviral property as well as used against hepatitis B and C viruses (Jacob et al., 2007). Its fruits are also used against murine norovirus-1 (MNV-1), feline calicivirusF9(FCV-F9), human norovirus and for curing fever (Kumar and Chauhan, 2008). The presence of gallic acid in this fruit is responsible for inhibiting the internalization of the virus into the cells and enhance its antiviral properties (Santhi et al., 2021).

\subsection{Other fruits}

Most of the fruits covered in this review are rich source of antioxidants, due to which they have high free radical quenching potential and help in boosting the immunity. Although, they are not contributing directly in COVID-19 or in viral diseases, but they have direct contribution in maintaining healthy life style. Thus, these fruits can be beneficial in quenching diseases, boosting immunity and reducing the risk of SARS infection.

Table 2: Importance of phytochemicals in disease quenching

\begin{tabular}{|c|c|c|c|c|c|c|}
\hline Sr No. & Fruit & $\begin{array}{l}\text { Phytochemical/functional } \\
\text { components }\end{array}$ & Key component & $\begin{array}{l}\text { Immunological } \\
\text { properties }\end{array}$ & Diseases & References \\
\hline 1. & Aegle marmelos & $\begin{array}{l}\text { Marmelide, Marmelosin, } \\
\text { Aegeline, Phenols (arbutin, } \\
\text { p-coumaric acid,caffeic acid, } \\
\text { cholorogenic acid, p-coum- } \\
\text { aroyl, protocatecheuic acid } \\
\text { and quinic acid), Flavonoids, } \\
\text { Xanthotoxol, Imperatorin }\end{array}$ & Marmelide & $\begin{array}{l}\text { Antiviral } \\
\text { Immunomodulatory } \\
\text { agents } \\
\text { Immunostimulant } \\
\text { Alpha-glucosidase } \\
\text { inhibitor } \\
\text { Anti-inflammatory } \\
\text { Antibacterial } \\
\text { Immune booster }\end{array}$ & $\begin{array}{l}\text { Diabetes } \\
\text { Diarrheal } \\
\text { diseases } \\
\text { Cancer } \\
\text { Ulcer } \\
\text { Cardiovascular } \\
\text { Gastrointestinal } \\
\text { disorders } \\
\text { Relaxed the } \\
\text { histamine- } \\
\text { induced } \\
\text { contractions }\end{array}$ & $\begin{array}{l}\text { Maity et al., } \\
2009 \text {; } \\
\text { Lambole et al., } \\
2010 \text {; } \\
\text { Patel and Asdaq, } \\
2010 \text {; } \\
\text { Sarkar et al., } \\
2020\end{array}$ \\
\hline
\end{tabular}




\begin{tabular}{|c|c|c|c|c|c|c|}
\hline 2. & $\begin{array}{l}\text { Artocarpus } \\
\text { lakucha }\end{array}$ & $\begin{array}{l}\text { Flavonoids, Tannins, Saponins, } \\
\text { Steroids, Glycosides, Triter- } \\
\text { penoids, Phenolic compounds, } \\
\text { Squalene, ß-amyrin acetate } \\
\text { and Lupeol acetate }\end{array}$ & B-amyrin acetate & $\begin{array}{l}\text { Antiviral } \\
\text { Anti HIV } \\
\text { Antiglycation } \\
\text { Anti-inflammatory } \\
\text { Antibacterial }\end{array}$ & $\begin{array}{l}\text { Diabetes } \\
\text { Prevent cellular } \\
\text { Aging } \\
\text { Coronary heart } \\
\text { disease } \\
\text { Cancer } \\
\text { Neurodegene- } \\
\text { rative diseases }\end{array}$ & $\begin{array}{l}\text { Hossain et al., } \\
2016\end{array}$ \\
\hline 3. & $\begin{array}{l}\text { Baccaurea } \\
\text { sapida } \\
\text { (Bermese grape) }\end{array}$ & $\begin{array}{l}\text { Ascorbic acid, Picrotoximaesin, } \\
\text { Romarinic acid, Gallic acid, } \\
\text { Salicyclic acid }\end{array}$ & Romarinic acid & $\begin{array}{l}\text { Anti-inflammatory } \\
\text { activity } \\
\text { Inhibit prostagland } \\
\text { in biosynthesis } \\
\text { Antiviral } \\
\text { Lower body } \\
\text { temperature } \\
\text { Analgesic } \\
\text { Cholesterol binding }\end{array}$ & $\begin{array}{l}\text { Rheumatoid } \\
\text { arthritis } \\
\text { Cellulitis } \\
\text { Jaundice } \\
\text { Regulate } \\
\text { immune } \\
\text { response }\end{array}$ & $\begin{array}{l}\text { Mann et al., 2015, } \\
\text { Gogoi, 2017; } \\
\text { Singh and Pandey, } \\
2021\end{array}$ \\
\hline 4. & $\begin{array}{l}\text { Berberois } \\
\text { aristata }\end{array}$ & Vitamin C & Ascorbic acid & $\begin{array}{l}\text { Anti-inflammatory } \\
\text { Wound healing }\end{array}$ & $\begin{array}{l}\text { Diabetes } \\
\text { Cancers } \\
\text { Diarrhea } \\
\text { Jaundice } \\
\text { Eye infection }\end{array}$ & $\begin{array}{l}\text { Sharma et al., } \\
2011\end{array}$ \\
\hline 5 & Bombaxceiba & $\begin{array}{l}\text { Phenols, Flavonoids, Tannins, } \\
\text { Saponins, Shamimicin, Lupeol, } \\
\text { Mangiferin, }\end{array}$ & Lupeol & $\begin{array}{l}\text { Hepato-protective } \\
\text { Hypotensive } \\
\text { Antiangiogenic } \\
\text { Antioxidant } \\
\text { activities } \\
\text { Diuretic }\end{array}$ & $\begin{array}{l}\text { Inflammatory } \\
\text { diseases } \\
\text { HIV }\end{array}$ & $\begin{array}{l}\text { Nagamani } \text { et al., } \\
2012\end{array}$ \\
\hline 6 & $\begin{array}{l}\text { Carissa } \\
\text { spinarum }\end{array}$ & $\begin{array}{l}\text { Alkaloids, Flavonoids, } \\
\text { Glycosides, Tannins, Alanine, } \\
\text { Carindone, Carinol, Carissone, } \\
\text { Carinol, Digitoxigenin, Lupeol, } \\
\text { Malonic and Glycolic acids, } \\
\text { Oxalic acid, Odoroside-H, } \\
\text { Phenyl alkaline, Vitamin C }\end{array}$ & Carindone & $\begin{array}{l}\text { Diuretic } \\
\text { Anti-inflammatory } \\
\text { Antiviral }\end{array}$ & $\begin{array}{l}\text { Malaria } \\
\text { Diabetes } \\
\text { Chest } \\
\text { complaints } \\
\text { Cough } \\
\text { Fever }\end{array}$ & $\begin{array}{l}\text { Ansari and Patil, } \\
2018 ; \\
\text { Berhanu et al., } \\
2020\end{array}$ \\
\hline
\end{tabular}




\begin{tabular}{|c|c|c|c|c|c|c|}
\hline 7 & $\begin{array}{l}\text { Cordia } \\
\text { dichotoma }\end{array}$ & $\begin{array}{l}\text { Arabinoglucon, Pyrrolizidine } \\
\text { alkaloids, Coumarins, } \\
\text { Flavonoids, Saponins, Terpenes, } \\
\text { Sterols, Quercetin, } \\
\text { Isorhamnetin, Cordioic acid, } \\
\text { Apigenin, Linolenic acid, } \\
\text { Hesperidin, Rutin, Arabinose, } \\
\text { Robinin, Caffeic acid, } \\
\text { Arabinoglucan }\end{array}$ & Arabinoglucon & $\begin{array}{l}\text { Antimicrobial activity } \\
\text { Hypoglycemic activity } \\
\text { Wound healing properties } \\
\text { Immune booster }\end{array}$ & $\begin{array}{l}\text { Fever } \\
\text { Cough }\end{array}$ & $\begin{array}{l}\text { Ganjare and } \\
\text { Raut, } 2019\end{array}$ \\
\hline 8 & $\begin{array}{l}\text { Celtis } \\
\text { australis }\end{array}$ & $\begin{array}{l}\text { Cyanidin-3,5-di-O-glucoside, } \\
\text { Pelargonidin-3,5-di-O- } \\
\text { glucoside, Epicatechin, Gallic } \\
\text { acid, Vanillic acid }\end{array}$ & Cyanidin-3,5-di-O-glucoside & $\begin{array}{l}\text { Antimicrobial activity } \\
\text { Lenitive and stomachic } \\
\text { properties } \\
\text { Immunity booster }\end{array}$ & $\begin{array}{l}\text { Colic } \\
\text { Amenorrhea } \\
\text { Dysentery } \\
\text { Diarrhea } \\
\text { Menstrual bleeding } \\
\text { Peptic ulcers }\end{array}$ & Ota et al., 2016 \\
\hline 9 & $\begin{array}{l}\text { Cornus } \\
\text { capitate }\end{array}$ & $\begin{array}{l}\text { Loganin, Morroniside and } \\
\text { Uroslic acid }\end{array}$ & Loganin & $\begin{array}{l}\text { Virus inhibitory activity } \\
\text { Antidiabetic }\end{array}$ & Diabetes & $\begin{array}{l}\text { He et al., 2016; } \\
\text { Bhatia et al., } \\
2019\end{array}$ \\
\hline 10 & $\begin{array}{l}\text { Crataegus } \\
\text { songarica }\end{array}$ & $\begin{array}{l}\text { Alkaloids, Terpenoids, } \\
\text { Flavonoids, Tannins, Phenolic } \\
\text { compounds, Saponins, Vitamin } \\
\text { C, Glycosides, Anthocyanidins }\end{array}$ & Anthocyanidins & $\begin{array}{l}\text { Cardio tonic } \\
\text { Dropsy } \\
\text { Diuretic }\end{array}$ & $\begin{array}{l}\text { Diarrhea } \\
\text { Slight phlegmasia } \\
\text { Tapeworm infections } \\
\text { Acute bacillary dysentery } \\
\text { Amenorhea hepatic disorders } \\
\text { Oxidative stress-related } \\
\text { diseases }\end{array}$ & $\begin{array}{l}\text { Hadi and Ibrar, } \\
\text { 2016; Mohan } \\
\text { and Midha, } \\
\text { 2017; Bhat } e t \\
\text { al., 2018 }\end{array}$ \\
\hline 11 & $\begin{array}{l}\text { Diospyros } \\
\text { lotus }\end{array}$ & $\begin{array}{l}\text { Ellagic acid, Gallic acid, } \\
\text { Methyl gallate, Myricetin-3- O- } \\
\alpha \text {-rhamnoside, Myricetin-3-O- } \beta \text { - } \\
\text { glucuronide, Quercetin }\end{array}$ & Gallic acid & $\begin{array}{l}\text { Antitussive } \\
\text { Sedative } \\
\text { Antiseptic } \\
\text { Antitumor } \\
\text { Laxative } \\
\text { Antidiabetic }\end{array}$ & $\begin{array}{l}\text { Diarrhea } \\
\text { Dry coughs, } \\
\text { Hypertension } \\
\text { Infections }\end{array}$ & $\begin{array}{l}\text { Rashed et al., } \\
2012 ; \text { Murathan, } \\
2020\end{array}$ \\
\hline 12 & $\begin{array}{l}\text { Elaeagnus } \\
\text { umbellate }\end{array}$ & $\begin{array}{l}\text { Vitamins A, C, E, Alkaloids, } \\
\text { Flavonoids, Steroids, Saponins, } \\
\text { Phenolic acids (cinnamic acid } \\
\text { and benzoic acid), Terpenoids } \\
\text { and Flavonoids } \\
\text { (epigallocatechin gallate, } \\
\text { myricetin), Phytoene, } \beta- \\
\text { carotene, Lutein, Phytofluene, } \beta \text { - } \\
\text { cryptoxanthin and } \alpha- \\
\text { cryptoxanthin }\end{array}$ & Epigallocatechin & $\begin{array}{l}\text { Reduce blood pressure } \\
\text { Coughs } \\
\text { Antiviral } \\
\text { Pulmonary infections }\end{array}$ & $\begin{array}{l}\text { Chronic diseases } \\
\text { Diabetes }\end{array}$ & $\begin{array}{l}\text { Gamba et al., } \\
2020\end{array}$ \\
\hline 13 & $\begin{array}{l}\text { Ficus } \\
\text { auriculata }\end{array}$ & 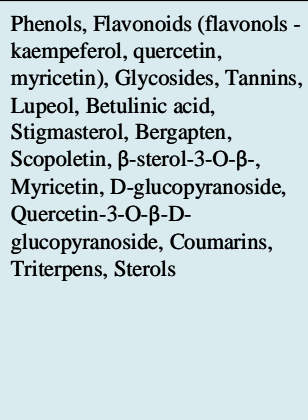 & $\begin{array}{c}\text { Quercetin-3-O-B-D- } \\
\text { glucopyranoside }\end{array}$ & $\begin{array}{l}\text { Hepatoprotective } \\
\text { Anticancerous } \\
\text { Antidiabatic } \\
\text { Hyperlipidemic } \\
\text { Hyperglycemic } \\
\text { Anti-inflammatory } \\
\text { Antioxidant } \\
\text { Antibacterial } \\
\text { Antifungal } \\
\text { Antiproliferative activities }\end{array}$ & $\begin{array}{l}\text { Inflammatory diseases } \\
\text { Diabetes } \\
\text { Cancer }\end{array}$ & $\begin{array}{l}\text { George et al., } \\
\text { 2016; Tamta } \text { et } \\
\text { al., 2021 }\end{array}$ \\
\hline
\end{tabular}




\begin{tabular}{|c|c|c|c|c|c|c|}
\hline 14 & Ficus palmate & $\begin{array}{l}\text { Alkaloids, Tannins, Flavonoids, } \\
\text { Terpenoids, Cardiac glycosides, } \\
\text { Germanicol acetate, Psoralene, } \\
\text { Bergapten, Vanillic acid, Glycoside } \\
\text { rutin, Coumarins, Furanocoumarin } \\
\text { glycosides, Isoflavones, Lignans }\end{array}$ & Germanicol acetate & $\begin{array}{l}\text { Demulcent } \\
\text { Emollient } \\
\text { Laxative } \\
\text { Poultice } \\
\text { Antitumor } \\
\text { Anti-inflammatory } \\
\text { Tonic medicament }\end{array}$ & $\begin{array}{l}\text { Gastrointestinal disorders } \\
\text { Hypoglycemia } \\
\text { Tumour } \\
\text { Ulcer } \\
\text { Diabetes } \\
\text { Hyperlipidemia } \\
\text { Fungal infections }\end{array}$ & $\begin{array}{l}\text { Joshi et al., } \\
\text { 2014; } \\
\text { Alqasoumi et } \\
\text { al., } 2014\end{array}$ \\
\hline 15 & $\begin{array}{l}\text { Fragaria } \\
\text { indica }\end{array}$ & $\begin{array}{l}\text { Phenolic acid, Flavonoids, Ascorbic } \\
\text { acid, Gallic acid, Catechin, }\end{array}$ & Catechin & Anti-inflammatory & $\begin{array}{l}\text { Treat abscesses } \\
\text { Boils and weeping eczema } \\
\text { Swellings }\end{array}$ & $\begin{array}{l}\text { Bhatt et al., } \\
2017\end{array}$ \\
\hline 16 & Juglans regia & $\begin{array}{l}\text { Flavonoids, Carotenoids, Alkaloids, } \\
\text { Nitrogen-containing compounds, } \\
\text { Polyphenolic, Tocopherol, Folate, } \\
\text { Melatonin, n-3 } \alpha \text {-linolenic acid } \\
\text { (ALA), Phenolic acids, Quercetin, } \\
\text { Tannins (glansrins A, B and C, } \\
\text { casuarinin, stenophyllarin) }\end{array}$ & Tocopherol & $\begin{array}{l}\text { Bronchodilator } \\
\text { Antihypertensive } \\
\text { Immunomodulatory } \\
\text { Antioxidant } \\
\text { Antidiabetic } \\
\text { Antimicrobial } \\
\text { Antiparasitic } \\
\text { Antiinflammatory } \\
\text { Antihistaminic }\end{array}$ & $\begin{array}{l}\text { Respiratory diseases } \\
\text { Cardiovascular diseases } \\
\text { Cancer } \\
\text { Immunological disorders } \\
\text { Analgesic } \\
\text { Gastrointestinal and endocrine } \\
\text { disease }\end{array}$ & $\begin{array}{l}\text { Jaiswal and } \\
\text { Tailang, 2017; } \\
\text { Al-Snafi, 2018 }\end{array}$ \\
\hline 17 & $\begin{array}{l}\text { Malus } \\
\text { baccata }\end{array}$ & $\begin{array}{l}\text { Quercetin (phloretin-2-xyloside, } \\
\text { quercetin-3-rhamnoside, quercetin- } \\
\text { 3-Gal/Glu, quercetin-3-Xyl/Ara), } \\
\text { Phloridzin, Phloretin, Procyanidins, } \\
\text { Catechin, Epicatechin, Cyanidin } \\
\text { glycosides, Cinnamic and Caffeic } \\
\text { acids, Chlorogenic acid, }\end{array}$ & Phloridzin & $\begin{array}{l}\text { Strengthening the immune } \\
\text { system } \\
\text { Antibiotic } \\
\text { Anticancer } \\
\text { Antimicrobial } \\
\text { Anti-inflammatory } \\
\text { Antioxidative }\end{array}$ & $\begin{array}{l}\text { Cancers } \\
\text { Cardiovascular diseases } \\
\text { Asthma } \\
\text { Diabetes } \\
\text { Obesity }\end{array}$ & $\begin{array}{l}\text { Kumari and } \\
\text { Dhaliwal, 2017; } \\
\text { Dadwal et al., } \\
\text { 2018; Petkova et } \\
\text { al., 2020 }\end{array}$ \\
\hline 18 & Morus alba & $\begin{array}{l}\text { Zeaxanthin, Ascorbic acid, } \\
\text { Carotene, Vitamin B1, Folic acid, } \\
\text { Folinic acid, Isoquercetin, } \\
\text { Quercetin, Tannins, Flavonoids, } \\
\text { Saponins, Zeaxanthin, Resveratrol, } \\
\text { Anthocyanins, Lutein, Morin, } \\
\text { Moracin }\end{array}$ & Carotene & $\begin{array}{l}\text { Immunomodulatory } \\
\text { Hypocholesterolemic } \\
\text { Antidiabetic } \\
\text { Antimicrobial } \\
\text { Antioxidant } \\
\text { Antistress } \\
\text { Antimutagenic } \\
\text { Anticancer } \\
\text { Anxiolytic } \\
\text { Anthelmintic } \\
\text { Nephroprotective } \\
\text { Hepatoprotective }\end{array}$ & $\begin{array}{l}\text { Obesity } \\
\text { Diabetes } \\
\text { Cancer } \\
\text { Cardiovascular diseases } \\
\text { Neurological disorders } \\
\text { Prevents certain damage to the } \\
\text { retina }\end{array}$ & $\begin{array}{l}\text { Devi et al., } \\
\text { 2013; Kadam et } \\
\text { al., } 2019\end{array}$ \\
\hline 19 & $\begin{array}{l}\text { Myrica } \\
\text { esculenta }\end{array}$ & $\begin{array}{l}\text { Myricitrin, Tannins (castalagin), } \\
\text { Phenolic acids, Flavonoids, } \\
\text { Terpenes, Glycosides, Steroids, } \\
\text { Volatile oils, Epigallocatechin 3-O- } \\
\text { gallate, Gallic acid, 3-O-galloyl- } \\
\text { epigallocatechin-( } 4 \beta \rightarrow 8) \text {-epigalloc- } \\
\text { atechin3-O-gallate, } \\
\text { Epigallocatechin-( } 4 \beta \rightarrow 8) \text { - } \\
\text { epigallocatechin3-O-gallate }\end{array}$ & Castalagin & $\begin{array}{l}\text { Antioxidant } \\
\text { Anticancer } \\
\text { Antidiabetic } \\
\text { Anti-inflammatory effects } \\
\text { Anxiolytic } \\
\text { Antibacterial } \\
\text { Antihelmintic } \\
\text { Antiallergic } \\
\text { Antimicrobial } \\
\text { Antiasthmatic }\end{array}$ & $\begin{array}{l}\text { COVID } 19 \\
\text { Asthma } \\
\text { Cough } \\
\text { Fever } \\
\text { Throat infection } \\
\text { Chronic bronchitis } \\
\text { Diarrhea } \\
\text { Ear and nose disorders } \\
\text { Body ache } \\
\text { Inflammation }\end{array}$ & $\begin{array}{l}\text { Kabra et al., } \\
\text { 2017; Kabra et } \\
\text { al., 2019; Patel } \\
\text { et al., 2021 }\end{array}$ \\
\hline
\end{tabular}




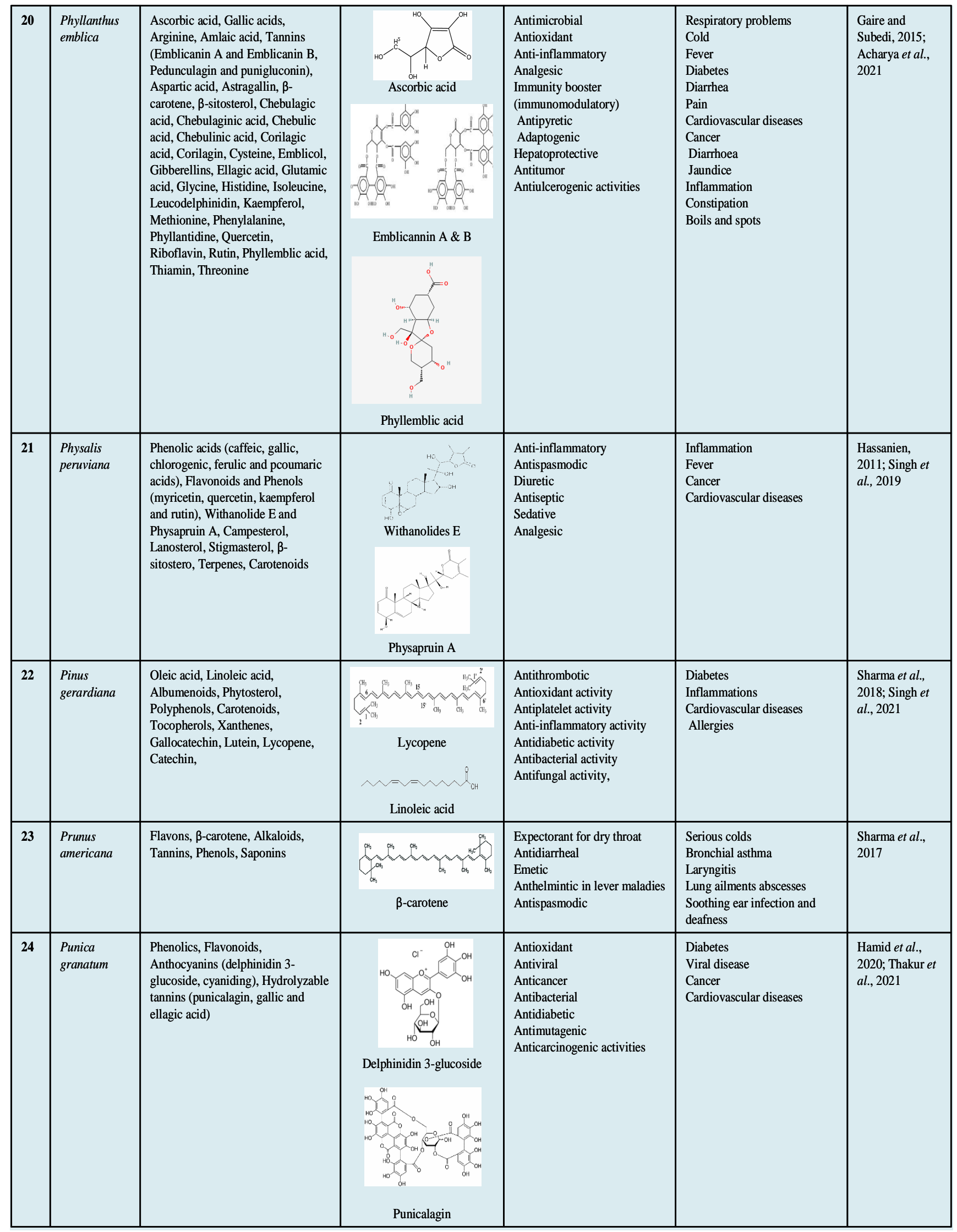




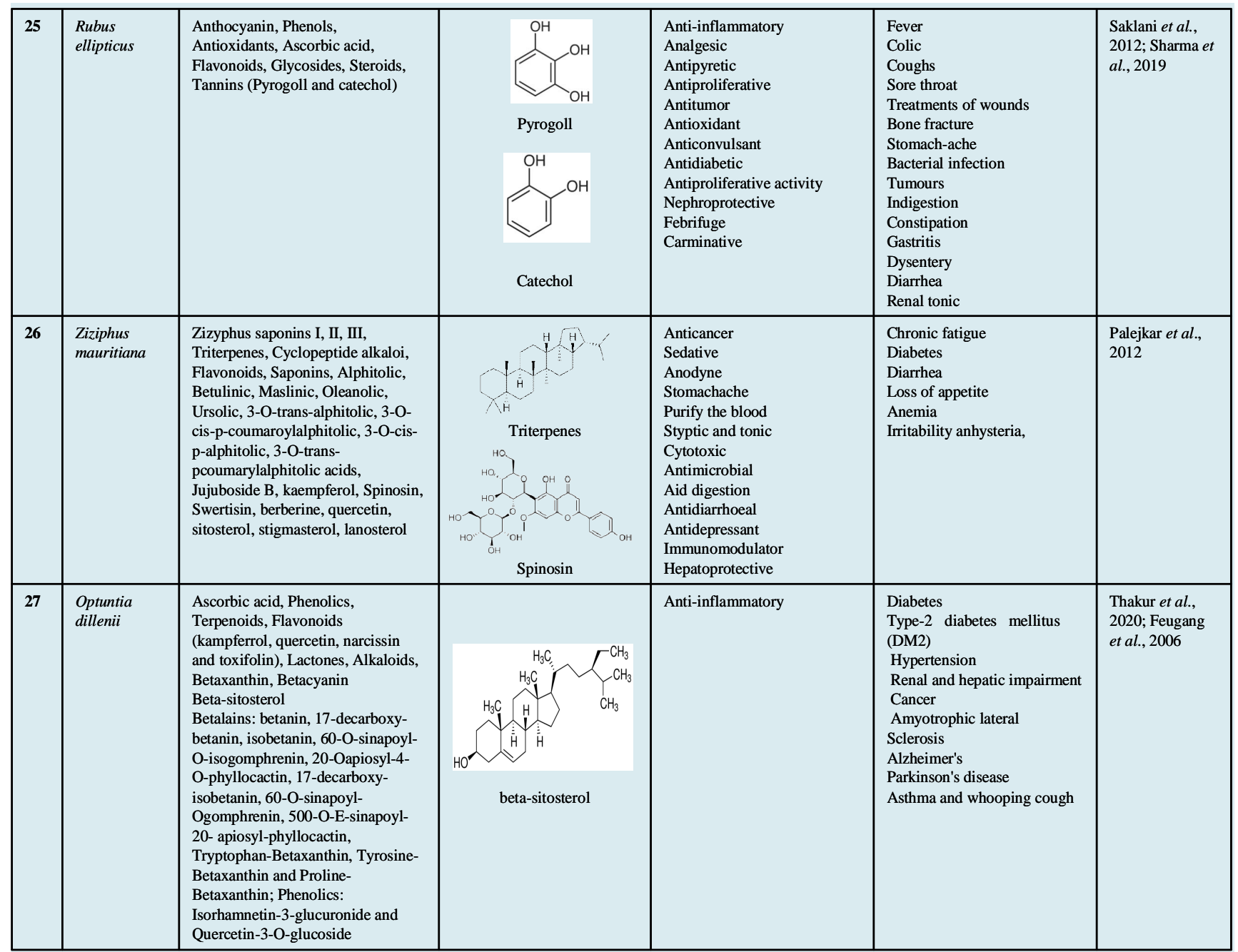

\section{Conclusion}

Current scenario says that the increasing threat of corona virus is not going to end soon and nobody knows who will be the next prey to this deadly virus. The re-occurring pandemic with new strains of virus every now and then has become a worldwide concern. We have vaccines but they are also strain specific however, the cocktail of drugs has been proven beneficial to some extent but with many side effects. Every doctor, nutritionist and health expert points on healthy food habits as major preventive agents of this virus like whole grains, fruits, vegetables and nuts. As we all know that indigenous fruits are potentially better than exotic ones because of their hardy nature and phytonutrients due to which they are used in "Ayurveda". But, these treasures of forests are being neglected due to ease of availability of exotic fruits and digitalization of food system. Some of these wild fruits are traditionally used against common cold, fever, diabetes, high blood pressure, etc., however, many of them are used against different types of viruses. This review stands affirm with the fact that use of wild fruits have the capability to boost the immunity of individuals against this ongoing pandemic as they are ocean of antioxidants.

\section{Conflict of interest}

The authors declare no conflicts of interest relevant to this article.

\section{References}

Acharya, C.K.; Khan, N.S. and Madhu, N.S. (2021). Medicinal uses of amla, Phyllanthus emblica L. (Gaertn.): A prospective review. Mukt Shabd Journal, 10(10):297-310.

Ahirvar, B.P.; Vishwakarma, R. and Chaudhry, S. (2017). Potentials and prospects of wild edible fruits in central India. International Journal of Current Advanced Research, 6(1):8561-8565.

Ahmad, B.; Hafeeza, N.; Raufb, A.; Bashirc, A.; Linfangd, H.; Rehmane, M.; Mubarakf, M.S.; Udding, M.S.; Bawazeerh, S.; Shariatii, M.A.; Dagliaj, M.; Wanl, C. and Rengasamy, K.R.R. (2021). Phyllanthus emblica: A comprehensive review of its therapeutic benefits. South African Journal of Botany, 138:278-310.

Ahmad, S.D.; Sabir, M.S.; Juma, M. and Asad, H.S. (2005). Morphological and biochemical variations in Elaeagnus umbellata Thunb. from mountains of Pakistan. Acta Botanica Croatica, 64(1):121-128.

Alqasoumi, S.I.; Basudan, O.A.; Al-Rehaily, A.J. and Abdel-Kader, M.S. (2014). Phytochemical and pharmacological study of Ficus palmata growing in Saudi Arabia Saudi. Pharmaceutical Journal, 22(5):460-471.

Al-Snafi, A.E. (2018). Chemical constituents, nutritional, pharmcological and therapeutic importance of Juglans regia: A review. Journal of Pharmacy, 8(11):01-21. 
Ansari, I. and Patil, D.T. (2018). A brief review on phytochemical and pharmacological profile of Carissa spinarum L. Asian Journal of Pharmaceutical and Clinical Research, 11(9):12-18.

Ashraf, Z.; Gani, A.; Shah, A.; Gani, A.; Noor, N.; Hassan, I. and Masoodi, F.A (2021). Bioactive compounds from plant sources as natural antivirals in combating RNA based viruses including COVID-19. Journal of Food Science and Nutrition, DOI: 10.24966/FSN-1076/100085

Berhanu, G.; Atalel, D. and Kandi, V. (2020). A review of the medicinal and antimicrobial properties of Carissa spinarum L. American Journal of Biomedical Research, 8(2):54-58.

Bhardwaj, K.; Silva, A.S.; Atanassova, M.; Sharma, R.; Nepovimova, E.; Musilek, K.; Sharma, R.; Alghuthaymi, M.A.; Dhanjal, D.S. and Nicoletti, M. (2021). Conifers phytochemicals: A valuable forest with therapeutic potential. Molecules, 26(10):1-38

Bhat, B.A.; Shergojri, F.A.; Gaur, M. and Shammi, Q.J. (2018). A review on Crataegus Songarica K. International Journal of Advance Research in Science and Engineering, 7(4):2425-2433.

Bhatia, A.; Singh, B.; Arora, R. and Arora, S. (2019). In vitro evaluation of the $\alpha$-glucosidase inhibitory potential of methanolic extracts of traditionally used antidiabetic plants. Complementary and Alternative Medicine, 19:74-77.

Bhatia, H.; Sharma, Y.P.; Manhas, R.K. and Kumar, K. (2018). Traditionally used wild edible plants of district Udhampur, J\&K, India. Journal of Ethnobiology and Ethnomedicine, https://doi.org/10.1186/s13002 018-0272-1.

Bhatt I.D.; Rawat, S.; Badhani, A. and Ranbeer, S.R. (2017). Nutraceutica potential of selected wild edible fruits of the Indian Himalayan region. Food Chemistry, 215:84-91.

Bhatt, K.; Thakur, N.S.; Thakur, A.; Hamid. and Sharma, C. (2020) Standardization of recipe for the preparation of wild jamun squash: Effect of packaging materials and temperature conditions on nutritional quality during storage. International Research Journal of Pure and Applied Chemistry, 21(12):34-44

Biswas, S.C.; Majumdar, M.; Das, S. and Mishra, T.K. (2018). Diversity of wild edible fruits used by the ethinic communities of Tripura, India. Indian Journal of Traditional Knowledge, 17(2):282-289.

Buddhisuharto, A.K.; Pramastya, H.; Insanu, M. and Fidrianny, I. (2021). An updated review of phytochemical compounds and pharmacology activities of Artocarpus genus. Biointerface Research, 11(6):1489814905 .

Cao, G; Sofic, E. and Prior, R. L. (1997). Antioxidant and prooxidant behavior of flavonoids: structure-activity relationships. Free Radical Biology and Medicine, 22:749-760.

Dadwal, V.; Agrawal, H.; Sonkhla, K.; Joshi, R. and Gupta, M. (2018) Characterization of phenolics, amino acids, fatty acids and antioxidant activity in pulp and seeds of high altitude Himalayan crab apple fruits (Malus baccata). Journal of Food Science Technology, 55(6):2160-2169.

Devi, B.; Sharma, N.; Kumar, D. and Jeet, K. (2013). Morus alba Linn: A phytopharmacological review. International Journal of Pharmacy and Pharmaceutical Sciences, 5:14-18.

Divya, N.; Nagamani, J.E. and Prabhu, S. (2012). Antioxidant and antihemolytic activities of Bombax ceiba pentandra spike and fruit extracts International Journal of Pharmacy and Pharmaceutical Sciences, 4(5):311-315

Fatima, A.; Singh, P.P.; Agarwal, P.; Irchhaiya, R.; Alok, S. and Verma, A. (2013) Treatment of various diseases by Carissa spinarum L.: A promising shrub. International Journal of Pharmaceutical Sciences and Research, 4(7):2489-2493.
Feugang, J.M.; Konarski, P.; Zou, D.; Stintzing, F.C. and Zou, C. (2006). Nutritional and medicinal use of cactus pear (Opuntia spp.) cladodes and fruits. Frontiers in Bioscience, 11:2574-2589.

Gaire, B.P. and Subedi, L. (2015). Phytochemistry, pharmacology and medicinal properties of Phyllanthus emblica Linn. Chinese Journal of Integrative Medicine, DOI: 10.1007/s1 1655-014-1984-2.

Gamba, G.; Donno, D.; Mellano, M.G.; Riondato, I.; Biaggi, M.D.; Randriamampionona, D. and Beccaro, G. L. (2020). Phytochemical characterization and bioactivity evaluation of autumn olive (Elaeagnus umbellata Thunb.) pseudo drupes as potential sources of health-promoting compounds. Applied Science. doi:10.3390/ app 10124354

Ganjare, A and Raut, N. (2019). Phytochemical and pharmacological properties of Cordia dichotoma (Bhokar): A short review. Asian Journal of Pharmacy and Pharmacology, 5(5):858-865.

George, M.; Joseph, L. and Paul, N.M. (2016). Ficus auriculata: A pharmacological update. International Journal of Current Research and Academic Review, 4(7):26-31

Giri, J.; Sathidevi, T. and Dushyanth, N. (1985). Effect of jamun seed extract on alloxan induced diabetes in rats. Journal of the Diabetic Association of India, 25:115-119.

Gogoi, B. (2017). Baccaurea ramiflora Lour.: Biochemical and ethnobotanical value with scope for bio-prospection. Annals of Plant Sciences, 6(7):1649-1652.

Goyal, M.; Nagori, B.P. and Sasmal, D. (2012). Review on ethnomedicinal uses, pharmacological activity and phytochemical constituents of Ziziphus mauritiana (Z. jujuba Mill). Spatula, 2(2):107-116.

Gyawali, R.; Paudel, P.N.; Basyal, D.; William, N.S.; Lamichhane, S.; Paudel,M.K.; Gyawali, S. and Khana, P. (2020). A review on ayurvedic medicinal herbs as remedial perspective for COVID-19. Karnali Academy of Health Sciences, 3:1-21.

Hadi, F. and Ibrar, M. (2016). Fruit plant diversity with special reference to their medicinal uses in the historical kalash valley, district Chitral, Hindukush range, Pakistan. Journal of Science and Technology, 40(1): 11-18.

Hamid; Thakur, N.S.; Thakur, A. and Kumar, P. (2020). Effect of different drying modes on phenolics and antioxidant potential of different parts of wild pomegranate fruits. Scientia Horticulturae, 274:109656 https:/ /doi.org/10.1016/j.scienta.2020.109656.

Hamid; Thakur, A. and Thakur, N.S. (2021). Role of functional food components in COVID-19 pandemic: A review. Ann. Phytomed., 10(1):240-250

Hamid; Thakur, N.S.; Kumar, P. and Thakur, A. (2017). Studies on preparation and preservation of ready-to-serve (RTS) beverage from underutilized mulberry (Morus alba L.) fruits and its quality evaluation during storage. International Journal of Current Microbiology and Applied Sciences, 6(9):1067-1079.

Hassanien, M.F.R. (2011). Physalis peruviana: A rich source of bioactive phytochemicals for functional foods and pharmaceuticals. Food Reviews International, 27:259-273.

He, K.; Song S.; Zou, Z.; Feng, M.; Wang, D.; Wang, Y.; Li, X. and Ye, X. (2016). The hypoglycemic and synergistic effect of loganin, morroniside, and ursolic acid isolated from the fruits of Cornus officinalis. Phytotherapy Research, 30(2):283-291.

Hossain, M.F.; Islam, M.A.; Akhtar, S. and Numan, S.M. (2016). Nutritional value and medicinal uses of Monkey Jack fruit (Artocarpus lakoocha). International Research Journal of Biological Sciences, 5(1):60-63. 
Jacob, J.R.; Mansfield, K.; You, J.E.; Tennant, B.C. and Kim, Y.H. (2007). Natura iminosugar derivatives of 1- deoxynojirimycin inhibit glycosylation of hepatitis viral envelope proteins. Journal of Microbiology, 45(5):431-440.

Jaiswal, B.S. and Tailang, M. (2017). Juglans regia: A review of its traditional uses phytochemistry and pharmacology. Indo American Journal of Pharmaceutical Research, 7(9):390-398.

Jalalpure, S.S. and Gadge, N.B. (2011). Diuretic effects of young fruit extracts of Bombax ceiba L. in Rats. Indian Journal of Pharmaceutical Sciences, 73(3):306-311.

Joshi, R.S.; Jagdale, S.S.; Bansode, S.B.; Shankar, S.S.; Tellis, M.B.; Pandya, V.K.; Chugh, A.; Giri, A.P. and Kulkarni, M.J. (2021). Discovery of potential multi-target-directed ligands by targeting host-specific SARS-CoV2 structurally conserved main protease. Journal of Biomolecular Structure and Dynamics, 39:3099-3114.

Joshi, Y; Joshi, A.K.; Prasad, N. and Juyal, D. (2014). A review on Ficus palmata (wild himalayan fig). The Journal of Phytopharmacology, 3(5):374-377.

Kabra, A.; Sharma, R.; Singla, S.; Kabra, R. and Baghel, U.S.B. (2017) Pharmacognostic characterization of Myrica esculenta leaves. Journal of Ayurveda and Integrative Medicine, pp:1-7.

Kabra, A.; Martins, N.; Sharma, R.; Kabra, R. and Baghel, U.S.B. (2019). Myrica esculenta Buch.-Ham. ex D. Don: A natural source for health promotion and disease prevention. Plants, 8:149. doi:10.3390/ plants 8060149 .

Kadam, A.R; Dhumal, N.D. and Khyade, V.B. (2019). The mulberry, Morus alba (L.): The medicinal herbal source for human health. International Journal of Current Microbiological Applied Science, 8(4):2941-2964.

Kasali, F.M.; Tusiimire, J.; Kadima, J.N.; Tolo. C.U.; Weisheit, A. and Agaba, A.G (2021). Ethnotherapeutic uses and phytochemical composition of Physalis peruviana L.: An overview. Scientific World Journal. https:/ /doi.org/10.1155/2021/5212348

Kaunda, J.S. and Zhang, Y.J. (2017). The Genus Carissa: An ethnopharmacological, phytochemical and pharmacological review. Natural Product and Bioprospecting, 7:181-199.

Khadka, D.; Dhamala, M.K.; Li, F.; Aryal, P.C.; Magar, P.R.; Bhatta, S.; Thakur, M.S.; Basnet, A.; Cui, D. and Shi, S. (2021). The use of medicinal plants to prevent COVID-19 in Nepal. Journal of Ethnobiology and Ethnomedicine. https://doi.org/10.1186/s13002-021-00449-w.

Kubola, J.; Siriamornpun, S. and Meeso, N. (2011). Phytochemicals, vitamin $\mathrm{C}$ and sugar content of Thai wild fruits. Food Chemistry, 126(3):972981

Kumar and Chauhan, S. (2008). Mulberry: Life enhancer. Journal of Medicinal Plant Research, 2(10):271-278.

Kumar, G.M.P and Shiddamallayya, N. (2016). Survey of wild edible fruits in Hassan forest division, Karnataka, India. Journal of Biodiversity and Environmental Sciences, 8(6):57-66.

Kumari, A. and Dhaliwal, Y.S. 2017. A study on nutritional composition and value addition of crab Apple (Malus baccata). American Journal of Food Science and Technology, 5(1):19-22.

Kutyauripo, I.; Chivheya, J.; Siyawamwaya, R. and Maguma, J. (2021). Food behavior towards natural functional foods during the COVID-19 pandemic. World Nutrition, 12(3):44-57.

Lambole, V.B.; Murti, K.; Kumar, U.; Sandipkumar, P.B. and Gajera, V. (2010) Phytopharmacological properties of Aegle marmelos as a potential medicinal tree: An overview. International Journal of Pharmaceutical Sciences Review and Research, 5(2):67-72.
Mahapatra, A.K.; Mishra, S.; Basak, U.C. and Panda, P.C. (2012). Nutrient analysis of some selected wild edible fruits of deciduous forests of India: An explorative study towards non-conventional bionutrition. Advance Journal of Food Science and Technology, 4(1):15-21.

Maity, P.; Hansda, D.; Bandyopadhyay, U. and Mishra, D.K. (2009). Biological activities of crude extracts and chemical constituents of bael, Aegle marmelos (L). Indian Journal of Experimental Biology, 47:849861 .

Manandhar, B.; Paudel, K.R.; Sharma, B. and Karki, R. (2018). Phytochemical profile and pharmacological activity of Aegle marmelos Linn. Journal of Integrative Medicine, 16(3):153-163.

Mann, S.; Sharma, A.; Biswas, S. and Gupta, R.K. (2015). Identification and molecular docking analysis of active ingredients with medicinal properties from edible Baccaurea sapida. Bioinformation, 11(9):437-443

Mohan, R. and Middha, D.R. (2017). Pharmacognostical and pharmacological profile of Crataegus songrica. International Journal of Interdisciplinary Research Centre, 3(3):2455-2275.

Monnerat, J.A.S.; Souza, P.R.; Cardoso, L.M.F.C.; Mattos, J.D.; Rocha, G.S. and Medeiros, R.F. (2020). Micronutrients and bioactive compounds in the immunological pathways related to SARS-CoV-2 (adults and elderly). European Journal of Nutrition, 60(2):559-579.

Murathan, Z.T. (2020). Phytochemical screening and antioxidant activity of Diospyros lotus L. Fruits grown in Turkey. Acta Scientarium Polonorum Hortorum Cultus, 19(2):49-55.

Nagamani, D.; Nagamani, J.E. and Prabhu, S. (2012). Antioxidant and antihemolytic activities of Bombax ceiba pentandra spike and fruit extracts. International Journal of Pharmacy and Pharmaceutical Sciences, 4(5):311-315.

Neag, M.A.; Mocan, A.; Echeverría, J.; Pop, R.M.; Bocsan, C.I.; Crişan, G. and Buzoianu, A.D. (2018). Berberine: Botanical occurrence, traditional uses, extraction methods, and relevance in cardiovascular, metabolic, hepatic, and renal disorders. Frontiers in Pharmacology, 9:557 doi:10.3389/fphar.2018.00557

Ota, A.; Visnjevec, A. M.; Vidrih R.; Prgomet Z.; Necemer M.; Hribar J.; Cimerman N. G; Mozina S.S.; Bucar-Miklavcic M. and Ulrih N.P. (2016). Nutritional, antioxidative and antimicrobial analysis of the Mediterranean hackberry (Celtis australis L.). Food Science and Nutrition, 5(1): 160-170.

Ozen, T.; Yildirim, K and Toka, M. (2017). The impacts of Elaeagnus umbellata Thunb. leaf and fruit aqueous extracts on mice hepatic, extrahepatic antioxidant and drug metabolizing enzymes related structures. Brazilian Journal of Pharmaceutical Sciences, http://dx.doi.org/ $10.1590 / \mathrm{s} 2175-97902017000317095$

Pal, M. (2020). Walnut: A highly nutritious food with several health benefits. Food and Nutrition, 3(1):118-121

Palejkar, C.J.; Palejkar, J.H.; Patel, A.J. and Patel, M.A. (2012). A plant review on Zizipus Mauritiana. International Standard Serial Number, 2(2):202-211.

Patel, B.; Sharma, S.; Nair, N.; Majeed, J.; Goyal, R.K. and Dhobi, M. (2021) Therapeutic opportunities of edible antiviral plants for COVID-19. Molecular and Cellular Biochemistry, 476:2345-2364.

Patel, P. and Asdaq, S.M.B. (2010). Immunomodulatory activity of methanolic fruit extract of Aegle marmelos in experimental animals. Saudi Pharmaceutical Journal, 18:161-165.

Petkova, N.; Ognyanov, M.; Inyutin, B.; Zhelev, P. and Denev. P. (2020). Phytochemical composition and antioxidant activity of Malus baccata (L.) Borkh. Fruits. Food Science and Applied Biotechnology, 3(1):47-55. 
Prajapati, S.K.; Kar, M.; Maurya, S.D.; Pandey, R. and Dhakar, R.C. (2017) Exploring phytochemicals and pharmacological uses of Cordia dichotoma (Indian cherry): A review. Journal of Drug Delivery and Therapeutics, 7(6):125-131.

Rashed, K.; Zhang, X.J.; Luo, M.T. and Zheng, Y.T. (2012). Anti-HIV-1 activity of phenolic compounds isolated from Diospyros lotus fruits. Phytopharmacology, 3(2):199-207.

Rusmadi, N.N.N.; Shahari, R.; Amri, C.N.A.C.; Tajudin, N.S. and Mispan, M.R. (2020). Nutritional value of selected edible ficus fruit in Kuantan. Journal of Tropical Life Science, 10(1):11-14.

Sahu, P.P.; Behera, L.; Nayak, S. and Samal, K.C. (2020). Health benefits of jamun (Syzygium cumini) an underutilised fruit: A ray in nanotechnology field. Journal of Pharmacognosy and Phytochemistry, 9(5):74-80.

Saklani, S.; Subhash Chandra, S.; Badoni, P.P. and Dogra, S. (2012). Antimicrobial activity, nutritional profile and phytochemical screening of wild edible fruit of Rubus ellipticus. International Journal of Medicinal and Aromatic Plants, 2(2):269-274.

Sallese, T.S.; Meneses, M.D.F.; Caldas, L.A.; Sa Guimaraes, T.E.; Oliveira, D.M. Jose,A.V.; Azevedo, R.C.; Kuster, R.M.; Soares, M.R. and Ferreira, D.F. (2021). Virucidal and antiviral activities of pomegranate (Punica granatum) extract against the mosquito-borne Mayaro virus. Parasites and Vectors, https://dx.doi.org/10.1186\%2Fs13071-02104955-4

Santhi, V.P.; Sriramavaratharaja, V.; Murugan, R.; Masilamani, P.; Shailendra, S.; Gurav; Sarasu, V.P.; Parthiban, S. and Ayyanar, M. (2021) Edible fruit extracts and fruit juices as potential source of antiviral agents: a review. Journal of Food Measurement and Characterization, 15:5181-5190.

Sarkar, T.; Salauddin, M. and Chakraborty, R. (2020). In-depth pharmacological and nutritional properties of bael (Aegle marmelos): A critical review. Journal of Agriculture and Food Research, https://doi.org/10.1016/j.jafr.2020.100081

Sasi, R. and Rajendran, A. (2012). Diversity of wild fruits in Nilgiri hills of the Southern Western Ghats: Ethnobotanical aspects. Internationa Journal of Applied Biology and Pharmaceutical Technology, 3(1):8287.

Shahrajabian, M.H.; Khoshkharam, M.; Zandi, P.; Sun, W. and Cheng, Q. (2019) Jujube, a super-fruit in traditional Chinese medicine, heading for modern pharmacological science. Journal of Medicinal Plants Studies, 7(4):173-178.

Shaikh, Z.; Sundarrajan, P.; Bhagtaney, L.; Zehra, S.; Zahra, K.; Badra, B.; Yigit, B.M.; Patel, N.; Alim, H.; Khan, J. and Ali, A. (2021). Applicability of vitamins in the management of COVID-19: An overview. Ann. Phytomed., 10(1):65-76.

Sharma, A.; Sharma, L. and Goyal, R. (2018). A review on himalayan pine species: ethnopharmacological, phytochemical and pharmacological aspects. Pharmacognosy Journal, 10(4):611-619.

Sharma, K.; Bairwa, R.; Chauhan, N.; Shrivastava, B. and Saini, N.K. (2011) Berberis aristata: A review. International Journal of Research in Ayurveda and Pharmacy, 2(2):383-388.

Sharma, M.; Kaura, J.; Kumar, V. and Sharma, K. (2019). Nutraceutical potential of Rubus ellipticus: A critical review on phytochemical potential, health benefits, and utilization. Think India Journal, 22(37):878-898.

Sharma, S.; Kaur, B.; Suttee, A.; Mukhtar, H.M. and Kalsi, V. (2017). Evaluation of antianxiety effect of dried fruits of Prunus americana Marsh. Asian Journal of Pharmaceutical and Clinical Research, 1:67-69.
Sharma, V.B.; Soni, M.K.; Onkar, J.M. and Sharma, O. (2019). Medicinal uses of jamun [Syzygium cumini (Linn) Skeeels.]: A review article. World Journal of Pharmaceutical and Medical Research, 5(8):89-90.

Shaygannia, L.E.; Bahmani, M.; Zamanzad, B. and Rafieian-Kopaei, M. (2015). A review study on Punica granatum. Journal of Evidence-Based Complementary and Alternative Medicine, 21(3):221-228.

Shin. T.; Wie, M.; Lee N.H.; Son, W.; Park, D.; Ahn, M. and Go, G. (2004). Functional bioactivity of Opuntia species. Oriental Pharmacy and Experimental Medicine, 4(4):219-226.

Singh, G.; Kumar, D. and Dash, A.K. (2021). Pinus gerardiana Wallichex. D. Don.: A review. Phytomedicine Plus. doi: https://doi.org/10.1016/ j.phyplu.2021.100024.

Singh, N.; Singh, S.; Maurya, P.; Arya, M.; Khan, F.; Dwivedi, D.H. and Saraf, S.A. (2019). An updated review on Physalis peruviana fruit: cultivational, nutraceutical and pharmaceutical aspects. Indian Journal of Natural Products and Resources, 10(2):97-110.

Soni, H. and Malik, J.K. (2021). Phytopharmacological potential of Zizyphus jujube: A review. Scholars International Journal of Biochemistry, 4(2):1-5.

Sooriyaarachchi, P.; Jeyakumar, D.T.; King, N. and Jayawardena, R. (2021). Impact of vitamin D deficiency on COVID-19. Clinical Nutrition, 44:372-378.

Tamta, G; Mehra, N. and Tandon, S. (2021). Traditional uses, phytochemical and pharmacological properties of Ficus auriculata: A review. Journal of Drug Delivery and Therapeutics, 11(3):163-169.

Thakur, A.; Joshi, V.K. and Thakur, N.S. (2019). Immunology and its relation with food components: An overview. International Journal of Food Fermentation and Technology, 9(1):1-16.

Thakur, A.; Thakur, N.S. and Kumar, P. (2017). Preparation of Myrica nagi (box myrtle) drink and effect of storage temperature on its quality. Journal of Applied and Natural Science, 9(4):2137-2142.

Thakur, A.; Thakur, N.S.; Hamid and Gautam, S. (2021). Effect of packaging on phenols, flavonoids and antioxidant characteristics of mechanical cabinet dried wild pomegranate (Punica granatum L.) arils. Journal of Applied and Natural Science, 13(1):101-109.

Thakur, N.S.; Chauhan, M. and Thakur, A. (2020). Studies on development and storage quality evaluation of betalains rich drink prepared from wild prickly pear (Opuntia dillenii haw) fruits. The Bioscan, 15(1):913 .

Umamaheswari and Chatterjee, T.K. (2008). In vitro antioxidant activities of the fractions of Coccinia grandis L. leaf extract. African Journal of Traditional Complementry and Alternative Medicine, 5(1):6173.

Wiersinga, W.J.; Rhodes, A.; Cheng, A.C.; Peacock, S.J. and Prescott, H.C. (2019). Pathophysiology, transmission, diagnosis, and treatment of coronavirus disease 2019 (COVID-19). Clinical Review and Education, 324(8):782-793.

World Health Organisation. (2020). https://www.who.int/emergencies/ diseases/novel-coronavirus-2019.

Yadav, V.K.; Singh, G.; Jhaa, R.K. and Kaushik, P. (2020). Visiting bael (Aegle marmelos) as a protective agent against COVID-19: A review. Indian Journal of Traditional Knowledge, 19:153-157.

Yadevendra, Y.; Sharma, A.; Sharma, U. and Sharma, K. (2020). Auspicious offering of lord shiva as a source of natural antiviral compounds against COVID-19: A review. Scholars International Journal of Traditional and Complementary Medicine, 3(7):131-139. 
Younus, I.; Fatima, A.; Ali, S.M.; Usmani, S.; Begum, Z.; Badar, S. and Asghar, R. (2016). A review of ethnobotany, phytochemistry, antiviral and cytotoxic/anticancer potential of Morus alba Linn. International Journal of Advanced Research and Review, 1(2):84-96.

Yuki, K.; Fujiogi, M. and Koutsogiannaki, S. (2020). COVID-19 pathophysiology: A review. Clinical Immunology, https://doi.org/10.1016/ j.clim.2020.108427
Zivkovic, I.; Savikin, K.; Zivkovic, J.; Zdunic, G.; Jankovic, T.; Lazic, D. and Radin, D. (2021). Antiviral effects of pomegranate peel extracts on human norovirus in food models and simulated gastrointestinal fluids. Plant Foods for Human Nutrition, 76:203-209.

Zulfqar, F.; Akhtar, M.F.; Saleem, A.; Akhtar, B.; Sharif, A. and Saleem, U. (2020). Chemical characterization, antioxidant evaluation, and antidiabetic potential of Pinus gerardiana (pine nuts) extracts. Journal of Food Biochemistry, DOI: $10.1111 / \mathrm{jfbc} .13199$.

Kanchan Bhatt, Sunakshi Gautam, Abhimanyu Thakur, N.S. Thakur, Hamid and Kritika Kaushal (2021). Role of wild fruits in combating COVID-19 infection: An overview. Ann. Phytomed., Volume10, Special Issue2 (COVID19): S128-S140. http://dx.doi.org/10.54085/ap.covid19.2021.10.2.15 